\title{
Correction to: 2019 update to: Management of hyperglycaemia in type 2 diabetes, 2018. A consensus report by the American Diabetes Association (ADA) and the European Association for the Study of diabetes (EASD)
}

\author{
John B. Buse ${ }^{1}$ - Deborah J. Wexler ${ }^{2,3} \cdot$ Apostolos Tsapas $^{4} \cdot$ Peter Rossing ${ }^{5,6} \cdot$ Geltrude Mingrone $^{7,8,9}$. \\ Chantal Mathieu $^{10}$. David A. D'Alessio ${ }^{11}$ - Melanie J. Davies ${ }^{12}$ \\ Published online: 15 May 2020 \\ (C) Springer-Verlag GmbH Germany, part of Springer Nature 2020
}

Correction to: Diabetologia.

https://doi.org/10.1007/s00125-019-05039-w

The incorrect figure legend was printed for Fig. 2. The correct legend should have read 'Choosing glucose-lowering medication in those with indicators of high-risk or established atherosclerotic cardiovascular disease (ASCVD), chronic kidney disease (CKD) or heart failure (HF)', as per the figure heading.

The online version of the original article can be found at https://doi.org/ 10.1007/s00125-019-05039-w

Melanie J. Davies

melanie.davies@uhl-tr.nhs.uk

1 Department of Medicine, University of North Carolina School of Medicine, Chapel Hill, NC, USA

2 Department of Medicine and Diabetes Unit, Massachusetts General Hospital, Boston, MA, USA

3 Harvard Medical School, Boston, MA, USA

4 Second Medical Department, Aristotle University Thessaloniki, Thessaloniki, Greece

5 Steno Diabetes Center Copenhagen, Gentofte, Denmark

6 University of Copenhagen, Copenhagen, Denmark
7 Fondazione Policlinico Universitario A. GerelliI RCCS, Rome, Italy

8 Università Cattolica del Sacro Cuore, Rome, Italy

9 Diabetes and Nutritional Sciences, King's College London, London, UK

10 Clinical and Experimental Endocrinology, UZ Gasthuisberg, KU Leuven, Leuven, Belgium

11 Department of Medicine, Duke University School of Medicine, Durham, NC, USA

12 Diabetes Research Centre, University of Leicester, Leicester General Hospital, Leicester, UK 